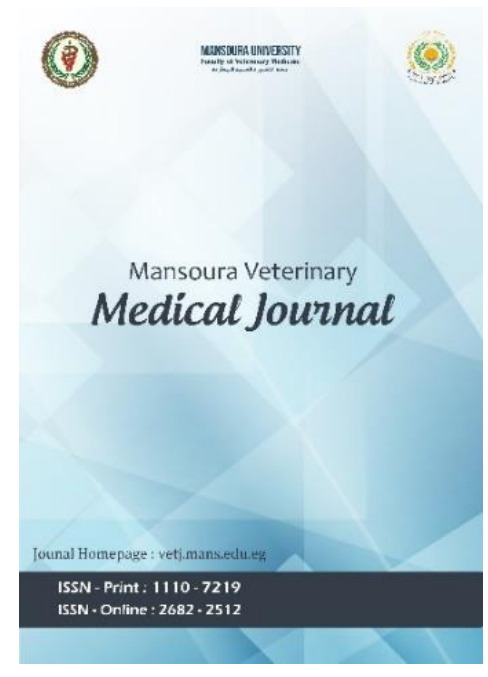

\title{
Genomic Overview into the Evolving Epidemiology of Methicillin-Resistant Staphylococcus aureus
}

Yara El dessouky, Shaimaa Mouftah, Mohamed Elhadidy

To cite this article: Yara El dessouky, Shaimaa Mouftah, Mohamed Elhadidy. Genomic Overview into the Evolving Epidemiology of Methicillin-Resistant Staphylococcus aureus. Mansoura Veterinary Medical Journal 2020; 21, 3: 125-131.

To link to this article: https://doi.org/10.35943/mvmj.202 0.21.322

Published online: 29 September 2020

Submit your article to this journal

(D) CrossMark data 


\title{
Genomic Overview into the Evolving Epidemiology of Methicillin-Resistant Staphylococcus aureus
}

\author{
Yara El dessouky ${ }^{1}$, Shaimaa F. Mouftah ${ }^{1}$, Mohamed Elhadidy ${ }^{1,2} \#$ \\ ${ }^{1}$ University of Science and Technology, Zewail City of Science and Technology, Giza, Egypt; \\ ${ }^{2}$ Faculty of Veterinary Medicine, Mansoura University, Mansoura, Egypt
}

ARTICLE HISTORY ABSTRACT
ART

Received: 10.09. 2020

Revised: 23.09 .2020

Accepted: 24.09 .2020

Address correspondences to Mohamed Elhadidy, University of Science and Technology, Zewail City of Science and Technology, Giza, Egypt +201220786861, E-mail: melhadidy@zewailcity.edu.eg

\begin{abstract}
Emerging infections represent an enormous challenge to both human and veterinary medicine. Identification of Methicillin-Resistant Staphylococcus aureus (MRSA) in various species and in food has raised concerns about the roles of animals in the epidemiology of MRSA. MRSA are a group of grampositive bacteria, distinct from other strains of $S$. aureus in that this pathogen is resistant to methicillin, oxacillin, and all beta-lactam antibiotics. The severity of infections caused by MRSA depends on the strain responsible for the infection and can vary from soft tissue infections to bacteremia and sometimes pneumonia. MRSA strains are divided into clones, based on their genetic makeup. According to the setting of infection, MRSA are divided into three epidemiological types: hospital acquired (HA-MRSA), community acquired (CA-MRSA), and livestock acquired (LA-MRSA) (ie. Transmitted from animal carriers). The epidemiology of HA-MRSA, CA-MRSA, and LA-MRSA is blurred as different recent genetic studies have revealed significant overlap of identical clones between HA-MRSA and CA-MRSA, and the significant increase of human infection caused by LA-MRSA. Furthermore, the animal-human and animal-animal transmission of LA-MRSA has prompted further investigation to study the origin of this epidemiological type and the transmission dynamics. The genetic and virulence profiles of different types of MRSA vary widely, where community acquired and livestock acquired strains are more virulent than hospital acquired strains. This review sheds light on three epidemiological groups of MRSA (HAMRSA, CA-MRSA, and LA-MRSA), and their most prevalent clonal clusters, that can consequently allow better understanding of their evolution, emergence, transmission, and global dissemination.
\end{abstract}

Keywords: Methicillin-Resistant Staphylococcus aureus, HA-MRSA, CA-MRSA, LA-MRSA, Epidemiology

\section{INTRODUCTION}

Methicillin-Resistant Staphylococcus aureus (MRSA) is one prominent cause of nosocomial infections. Up until the 1960s, MRSA infections were hospital acquired and highly prevalent between individuals who have been admitted to an intensive care unit or nursing homes [1]. In 1980, the first communityacquired MRSA infection (CA-MRSA) was identified in the US [1], with a steadily increasing prevalence of infection in adults and children in the decades following its discovery. The CDC defines CA-MRSA as a MRSA infection in a patient with no history of any of the predisposing factors, who has not been hos pitalized or admitted to a nursing home in the previous year [2]. All MRSA types are resistant to oxacillin, methicillin and all beta-lactam antibiotics, with some strains being multi -resistant to other classes of a ntibiotics as well [2-4]. Resistance to all $\beta$ lactam antibiotics is mediated by the mecA gene that is located on a genetic island known as the Staphylococcal Cassette
Chromos ome (SCC) [3,5]. The mecA gene codes for a n alternate peni cillin binding protein (PBP) known as PBP2a. This modified transpeptidase has low affinity to the beta-lactam ring found in all antibiotics of the $\beta$-lactam class and binding of this protein to the ring makes the bacteria resistant $[2,5,6]$. Predisposing risk factors associated with MRSA infections include recent hospitalization or surgery, intravenous drug use, presence of any indwelling medical device that passes through the skin to the body, dialysis, close contact with a MRSA infected patient, low socioeconomics tatus, crowdedness, malignancy or misuse of antibiotics [1-6].

Annually, a round 1.2 million people acquire invasive MRSA infections during hospitalization, resulting in MRSA to account for $20 \%$ of all nosocomial infections [7]. According to the CDC report in 2017, around 120,000 S. aureus infections and 20,000 associated deaths were recorded in the US alone [8]. Additionally, the WHO priority pathogen list for 2017 reported 
MRSA as a high priority pathogen (level 2) [9], des pite the fact that the numbers of MRSA infections have been decl ining over the past few years [7]. In addition to the drastic consequences of a MRSA infection on individuals, healthcare facilities are often financially challenged by the rather high prevalence rates of MRSA. A US study a cross 55 hospitals es timated the cost per MRSA infection to be $\$ 12,197$ while other studies in France indicate a mean cost per infection of $\$ 39,500$ [10]. Even a mong lower-middle-income developing countries, the total health expenditure in Egypt is rather insufficient. Only $4.75 \%$ of GDP is spent on providing inadequate healthcare to citizens [11], which highlights the urgency of enhancing our knowledge of the organism and designing plans to combat its spread.

In Egypt, reports have described a prevalence rate of 50$82 \%$ a mong hos pitalized patients in Ca iro and Al exandria but a lower rate of $24 \%$ in Minia [12]. Other reports of community acquired MRSA (CA-MRSA) indicated a prevalence rate of 19$47 \%$ [1]. It has also been reported that $S$. aureus isolates are primarily responsible for wound and surgical site infections at Minia University Hospital [13]. However, the lack of a national surveillance system makes it challenging to extrapolate the data from the separate cities to give nation-wide data.

The aim of this review is to provide a conclusive overview of different epidemiological groups of MRSA (HA-MRSA, CAMRSA and LA-MRSA), highlighting the possible threat of these groups on the Egyptian population. This is in accordance with the national multi-sectorial plan of the World Antibiotic Awareness week forum [14] held in Cairo University in November 2017, which entails; 1) increasing awareness of antimicrobial resistance through trainings, education and communication 2) improving incidence of infection via better prevention and control methods 3 ) monitoring and optimizing the use of antibiotics in animal and human health, and 4) encouraging and supporting research and innovation to strengthen knowledge nation-wide.

\section{Application of Multi-locus sequence typing to study the clonal population structure of MRSA}

Multilocus sequence typing (MLST) is a typing technique that is used to identify and characterize bacteria una mbiguously, usually based on fragments of 7 house-keeping genes [15-18]. A DNA sequencer is us ed to sequence around 450-500 base pairs of the internal fragments of each of these genes [15]. Different sequences (polymorphisms) found in each gene within a species are identified as different alleles and those different a llel es are then defined as a s equence type (ST) [15-17]. Therefore, 7 integers correspond to the al leles found at the 7 loci of the house-keeping genes that were sequenced $[16,17]$. Different sequences in the same isolate are given different allele numbers regardless of how different they are (ie. whether they differ in one or more nucleotide) [17-19]. This process usually gives billions of different allele profiles because most bacterial species exhibit enough variation within those genes, providing several alleles per locus. The allelic profile is defined by the alleles found at each locus. Given that many potentia l a lleles can be found at each locus, it is highly unlikely that identical allelic profiles exist by chance [18]. Isolates with the sa me allelic profiles are classified as members of the same clonal complex (CC). Strains that share almost identical allelic profiles are referred to as sequence types while those that share only some of the alleles are related and therefore referred to as clonal complexes $[15,19]$. MLST is usually used to investigate the serotypes, geographical origins and relatedness of different strains without establishing phylogenetic relationships (ie. they only show descent and emergence but complementary sequence information is needed to establish phylogenetic relationships) $[15,19]$. All MLST data that were collected have been grouped into one online library, publicly available at http://www.pubmlst.org [17].

MLST has been previously used in different epidemiological studies as an efficient tool of monitoring the evolution and changes in the population structure of microbes, especially pathogens, in epidemiological studies [19]. MLST has several advantages including high reproducibility, portability, easy interpretation of data and that access to live bacterial isolates is not required $[17,19]$.

\section{Genetic insights into HA-MRSA and CA- MRSA}

Differences in the SCCmec type are used to distinguish between HA-MRSA and CA-MRSA. HA-MRSA strains usually have SCCmec types I, II and III while CA-MRSA have the s maller types IV and V [3,6]. A significant percentage of CA-MRSA carry genes for the exotoxin Panton-Valentine leukocidin (PVL) which is lethal to leukocytes $[3,20-25]$. Genetic and molecular studies support the hypothesis that PVL genes, along with other exotoxins, are res ponsible for the enhanced virulence of CAMRSA over HA-MRSA, leading the former to cause necrotic skin lesions and necrotizing pneumonia more frequently $[3,4]$. Multiple insertion sequences were identified in the mec region of CA-MRSA and are speculated to contribute to the resistance of multi-drug resistant (MDR) strains against quinolones, clindamycin, erythromycin, trimethoprim, and gentamicin $[5,26,27]$. Severe invasive disea ses are more as sociated with CA-MRSA than HA-MRSA, as CA-MRSA was found to infect heal thy young individuals, possibly with no identified risk factors or comorbidities $[3,28]$. CA-MRSA is predominantly is olated from skin and soft tissue infections (SSTIs), which are usually mild but can occasionally result in hospitalization and mortality $[1,3,28]$. Despite the above differences and the distinct phenotypical features of both strains, HA-MRSA and CA-MRSA a re becoming increasingly difficult to distinguish from one another. As reported in [28], cl onal complexes CC1, CC5, CC8 (ST8), CC22, CC30 (ST30), and CC59 (ST59) have been identified in isolates from community and healthcare settings $[23,24]$. 


\section{Clonal relation between CA-MRSA and LA- MRSA}

MRSA isolates of animal origin are referred to as livestock acquired (LA). Cl onal complexes CC8, CC15, CC22, CC30, CC45, CC80, and CC152 are mainly as sociated with human isolates [29-33,34]. Of CCs isolated from domestic animals, CC130 and CC398 have low host specificity and a re therefore frequently isolated from human patients [22-24,29,33,35]. Furthermore, CC97, CC133, CC522 and the lineage ST151 a re mainly found in ruminant isolates while the lineage ST385 is present in poultry is olates $[23,29,34,36]$. Other clonal lineages such as CC1, CC5, CC59, CC9, ST425 were identified, some of which were i solated from companion animals such as dogs, cats, and horses [22$25,29,35,36]$.

Comparative analyses of CC398 revea led that even though it currently represents poultry and pig isolates, it originally descended from an ancestral population in humans while ST91 originated from ruminants $[33,34,37]$. The two most widely disseminated lineages in livestock are CC398 and CC9 $[5,6,19,23,24,29]$. CC398 currently includes 43 different sequence types such as ST541, ST1965, ST1966, ST1967, and ST1968, of which ST398 is the main colonizer in pigs [22,23,33$36,38]$.

Marked genetic s imilarities were found between CA-MRSA and LA- MRSA as both clades share the lineages ST239 and ST398, both contain SCCmec IV and V, while exhibiting little differences in their staphylococcal protein a (spa) types [22$24,35,37,39]$. This suggests that dissemination occurs in a bidirectional manner; from animals to humans and vice versa, with specifically high prevalence of LA-MRSA between veterina rians, farmers and those in close contact with animals $[23,25,39]$.

Growing evidence currently suggests an overlap between CA- and LA-MRSA exists [22]. It was found in a recent study that MRSA ST398 isolated from slaughter pigs, bovine mastitis and farm pers onnel shared the same genetic virulence gene profiles $[20,22-24,30,36,40]$. Some CC398 isolates of human origin are more virulent than others due to the production of an alphatoxin and the ability to lyse human polymorphonuclear leukocytes $[6,40]$. In a ddition, in Japanese pigs, CA-MRSA strain ST9 was identified [41].

SCCmec usually contain other resistance genes and genes responsible for combating harmful chemicals such as heavy metals [6]. MRSA strains can acquire this genetic island by means of horizontal gene transfer [6] $[20,21,26]$. A primitive species of staphylococci, S. scuiri, us ually found on the skin of domestic animals has been suggested to be the species of origin for the evolution of SCCmec $[6,40]$. This is supported by findings that the PBP2a of MRSA is very similar to the na tive PBPs of $S$. scuiri [6]. Some studies suggest that mecA was already present in primitive $S$. aureus gene pools even before the introduction of methicillin, given the rapid emergence and prevalence of
MRSA and the observation that key events in the evolution of the mecA gene were found to be directly linked to time points when antibiotics were introduced in veterinary and human clinical settings $[6,42]$. The mecA1 is a ubiquitous homologue to $m e c A$ and was identified in primitive $S$. scuiri, sugges ting that the former is the precursor of the mecA gene $[6,42]$. This is supported by the finding that certain strains resistant to $\beta$ lactams possess this quality due to altered promoter region of that gene $[42,43]$. Further evidence that supports the hypothesis is that mecA1 gene was able to exhibit $\beta$-lactam resistance when experimentallyinduced into an $S$. aureus strain by producing a protein with similar properties to MRSA PBP2a [42].

One interes ting finding is that antibiotic resistance levels of clinically isolated MRSA are wi dely variable [6] $[20,26]$, as some MRSA strains were only very slightly resistant to methicillin whil e other strains were highly resistant. Evidence suggests that el evated resistance levels may be attributed to el evated levels of expression of PBP2a, by up-regulation of the mecA gene [6]. Other studies propose that auxiliary factors, such as cell division protei ns, known as the "femfactors" a lso determine resistance and virulence [4] [44]. Nonetheless, the exact mechanism by which resistance levels vary so widely is poorly understood. Further investigations might open new windows for treatment of MRSA [4]. In addition, whole genome sequencing (WGS) of the 2 predominant lineages; CA-MRSA ST93-IV and LA-MRSA ST398-V, suggests that anthropozoonotic and zoonotic transmissions occurred and that the movement of ST93-IV from humans to pigs then back to humans, in presence of the MDR ST398-V strain, has led to the acquisition of multiple antibiotic resistance determinants to tetracycline, clindamycin and chloramphenicol $[23,25,32,45]$. Such determinants were origi nally absent from ST93-IV [45]. Another study suggests that a host jump of CC398 occurred from humans to animals, followed by acquisition of the SCCmec [38].

A study conducted by Huijsdens et al in 2006 found a possible correlation between pig-farming and contracting MRSA in the Netherlands [6]. Using pulsed field gel el ectrophoresis (PFGE) with the restriction enzyme Smal and $S$. aureus spa typing for identification, it was found that pig-MRSA is olates were also found in pig-farmers and their families with no known history of exposure to predisposing risk factors $[4,38]$. This strain of LA-MRSA was not found in other non-pigfarming individuals of the same community [4]. What is even more alarming is the finding that those identified PFGE nontypeable (ie. Livestock acquired) MRSA strains are not only transmitted from pig to human but from human to human as well $[4,38]$.

Another study that demonstrates the ease of transmission of LA-MRSA was conducted by Voss et al, [45]. This study found a 760-fold higher MRSA carriage rate among pig farmers compared to the general Dutch population. A direct correlation 
was found between the proportion of MRSA-positive a nimals, intensity and duration of human-to-animal contact, and CC398 human colonization [45]. Mutters et al. [41] and Sa hibzada et al. [45] found that CC398 can survive in the environment, which contributes towards further dissemination.

The most prominent clonal complexes of LA-MRSA are CC398, CC9, CC97, among other lineages. One of the most prominent virulence factors of LA-MRSA CC 398 is its ability to acquire foreign DNA, such as the PVL gene $[38,45]$. Although contrary to information reported in [39], CC398 that produce enterotoxins, adhesion factors, proteases and superantigenlike proteins have also been identified in pig, poultry, and bovine samples $[38,45]$. Another piece of evidence that supports that CC398 might have been of human origin is the finding that certain strains carry immune eva sion cluster (IEC) $[21,22,27,33,38]$. This cluster is usually absent in animal isolates [38]. CC9 is the major LA-MRSA strain in As ia and was first identified in Europe in 2008 [38]. However, the main strain in Europe is ST398. CC9 strains are generally MDR and carry genes against lincosamides and PHLOPSA (drug classes phenicol, lincosamides, oxazolidinones, pleuromutilins and streptogramin A) [38]. CC97 is a gl obal leading cause of bovine mastitis that is occasionally found small ruminants, pigs, and humans $[22,23,32,36,38]$. It is suggested that a host jump occurred from bovine to human subjects around 40 years ago [38]. Several other lineages of LA-MRSA have been identified in Africa such as the human-associated ST5-SCCmecIV and ST88SCCmec IV in pigs from Senegal, and ST153-SCCmec NT from healthy sheep in Tunisia [38]. Human-associated $S$. aureus lineages have also been identified in chimpanzees, possibly due to humanosis $[4,38]$.

\section{Evolving epidemiology of MRSA}

Several factors influence prevalence of MRSA such as geographic area, a nimal origin and the environment ha rboring the human-animal interaction. The most prevalent strain in Europe and USA is CC398 while that in Asia is CC9 [38]. Aquatic products, raw beef and poultry, pork and dairy products are all possible food-borne sources of infection $[35,46]$. CC398 has als o been infrequently identified in horses a nd veal calves [38]. Contracting MRSA from any of the a bove sources can occur by consumption or poor handling of contaminated sources $[29,38]$. Egypt is the largest African dairy producer and among the lea ding cheese producers in the Mediterranean region, with household a nimals producing around $80 \%$ of cow and buffalo milk [47]. Being a developing country, safety practices neces sary to limit tra nsmission of MRSA via dairy products are usually overlooked which poses considerable threat to the public health [47].

MRSA clones capable of colonizing several livestock ani mals at once have also been identified that are capable of infecting humans either via direct contact or consumption of their conta minated products (meat, milk, etc.) by humans [39]. A study conducted in 2019 in China revealed that $7.4 \%$ of $S$. aureus isolated from contaminated food were MRSA while in [29], 75\% of isolates from broiler-chicken were LA-MRSA. In the same study, the prevalence rates of LA-MRSA in turkey, turkey meat, broilers, and chicken meat were $36 \%, 13 \%, 5 \%$, and $5 \%$ respectively. In another study, two chicken samples tested posi tive for MRSA s trains USA300 and USA500 [29]. Both strains are CA-MRSA which suggests a host jump occurred from humans to the chickens. Astudy by Mutters et al. [41] estimates that $85 \%$ of all farms had MRSA-positive a nimals. Another study provided further evidence that suggests that the MRSA strain CC398 can be transmitted between humans by direct contact [40]. In that study, isolated CC398 were divided into two different clades; a pig clade and a humanclade. Among the pig clade, it was found that some isolates originated from humans without pig contact, which supports the hypothesis that CC398 could be transferred via human-to-human contact [40]. CC398 caus es a wi de spectrum of illnesses in humans that ranges from minor to invasive infections that may require hospitalization. The low prevalence of CC398 a mong humans not in usually in frequent contact with animal res ervoirs can be attributed to the low transmissibility of the strain, which lowers the clinical significance of CC398 infections [45]. ST398 is a more threatening strain because despite being less transmissible than other strains, it is co-resistant to non $\beta$-lactam a ntibiotics used in medical and veterinary practice [45]. Poor hygienic practices between individuals working in animal husbandry and food processing, along with improper storage are the primary risk factors in food-borne MRSA transmission $[29,38,40]$. This poses major health risks because some LA-MRSA produce multi-drug resistant enterotoxins $[29,39,40]$.

\section{Key epidemiological differences between CA-MRSA, HA-MRSA and LA-MRSA}

Though epidemiological and molecular typing techniques have discriminated MRSA into HA, CA and LA, overlaps between these three epidemiological groups, due to convergent and divergent evolution, are observed. For example, nosocomial infections by CA-MRSA s trains were identified, LA-MRSA strains were isolated from hospital environments and LA- and HAstrains were i solated in community a cquired infection setting [39]. In other words, isolates from different sources with the same ST were identified [20,21,26,27,39]. Recently, evidence is accumulating that CA-MRSA strains can shuttle between hospitals and the community, suggesting that such strains might replace the tra ditional, often less virulent, HA-MRSA in heal thcare settings $[23,24,32,39]$. Another interesting finding is that HA-MRSA strains investigated in this study were more resistant but less virulent, contrary to CA- and LA-strains which are usually more virulent but more susceptible to antibiotics [39]. A recent study highlighted the association between human infections and MRSA ST398 in the general population 
[39]. Evidence also suggests that the genetic diversity of MRSA is correlated wi th the ra pid s pread of CA-MRSA, which acts as a genetic carrier between HA-MRSA and LA- MRSA [39].

\section{Application of genomics in understanding the evolving epidemiology of MRSA}

Molecular typing is of utmost significance in tracking emergence, spread and evolution of pathogens, assessing efficacy of outbreak control measures and identifying possible origins of outbreak [48]. Though conventional targeted molecular typing methods, such as pulsed-field gel el ectrophoresis (PFGE) and MLST can provide useful insight into the epidemiology of a certain pathogen, Whole Genome Sequencing (WGS) significantly enhances resolution $[48,49]$. This is because the latter negates the as sumption that isolates with similar genotypes that are linked by epidemiological data represent linked cases of infection [48]. In other words, in MLST, it is usually assumed that isolates with similar genotypes have similar epidemiology which is often misleading, especially with the observed overlap between different clones of the different sources of MRSA. However, it should be noted that whol egenome and core genome MLST schemes have enabled comparison of test sequences with large curated sets of predefined genes for a particular species [48]. With WGS readily available on a large scale, a more detailed study of patterns of spread, transmission and evolution of MRSA is now available $[48,49]$. It is predicted that with the continuously decreasing costs of WGS and wider availability of the technology, WGS will no longer be confined within resea rch and reference laboratory facilities but will be available in routine clinical labs to identify infection, prevention and control tactics in real time [48]. To highlight the significance and accuracy of WGS in MRSA detection, Humphreys and Coleman [48] reported a study where isolates from 17 pediatric MRSA patients in a neonatal unit outbreak were typed using PFGE, spatyping and WGS. The study reported that using WGS, 12 of the 17 isolates were genetically unique and therefore could not have contributed to the outbreak [48]. This finding was critical in such case as it suggests that hospital personnel abided by infection prevention methods [48]. The finding that all isolated strains were USA300 suggests that multiple independent introductions of the strain have occurred [48]. WGS has also been us ed to investigate commonality, colonization and spreading of CA-MRSA strains $[48,49]$. The authors in $[48]$ stress that WGS has significantly expanded our understanding of the epidemiological features of HA- and CA- MRSA, evolution patterns of resistance and trans mission $[44,48]$. WGS can confirm low, usually undetected, level ongoing clusters that could be cross-transmitted, as is the case with LA-MRSA $[48,49]$. The available data can provide guidance for designing infection control schemes to prevent future outbreaks.

\section{Conclusion}

MRSA infections can often go undiagnosed because the skin lesions caused resemble spider bites. This leads to an underestimation of the number of CA-MRSA cases. The increasing overlap between different strains and origins of MRSA makes it even more challenging to distinguish between the strains and therefore design suitable action plans to combat infection in the variable settings and strongly advice for applying One Health a pproach to track MRSA, especially LAMRSA. Although currently uncommon in community and hospital settings, immediate monitoring of the prevalence of the strain ST398 is necessary to avoid outbreaks [39]. Lack of conclusive data in Egypt and the gap created by the individual, unlinked and unrelated studies make it difficult to draw accurate conclusions.

\section{Conflict of Interest}

All authors declare no conflict of interest.

Ethical approval: Not required.

\section{Authors' contribution}

Y.E. wrote the paper with some additional edits and supervision from S.F.M and M.E.

\section{REFERENCES}

[1] Gorak, E., Yamada, S. and Brown, J., 1999. Community-Acquired MethicillinResistant Staphylococcus aureus in Hospitalized Adults and Children without Known Risk Factors. Clinical Infectious Diseases, 29(4), pp.797-800. https://doi.org/10.1086/520437

[2] Laboratory Testing | MRSA | CDC [Internet]. Cdc.gov. 2019 [cited 2020 Aug 29]. Available from: https://www.cdc.gov/mrsa/lab/index.html

[3] Bukharie HA. A review of community-acquired methicillin-resistant Staphylococcus aureus for primary care physicians. J Family Community Med. 2010;17:117-20. https://doi.org/10.4103/1319-1683.74320

[4] Huijsdens XW, van Dijke BJ, Spalburg E, van Santen-Verheuvel MG, Heck MEOC, Pluister GN, et al. Community-acquired MRSA and pig-farming. Ann Clin Microbiol Antimicrob. 2006;5:26. https://doi.org/10.1186/1476-0711$5-26$

[5] Herold BC, Immergluck LC, Maranan MC, Lauderdale DS, Gaskin RE, BoyleVavra S, et al. Community-acquired methicillin-resistant Staphylococcus aureus in children with no identified predisposing risk. JAMA. 1998;279:593-8. https://doi.org/10.1001/jama.279.8.593

[6] Vestergaard $M$, Frees $D$, Ingmer $H$. Antibiotic resistance and the MRSA problem. Microbiol Spectr [Internet]. 2019;7(2). http://dx. doi.org/10.1128/microbiolspec.GPP3-0057-2018

[7] Pal S, (Pharm) BS, MBA, PhD Professor of Pharmacy Administration College of Pharmacy, Health Sciences, St. John's University Jamaica, et al. Trends in MRSA Prevalence [Internet]. Uspharmacist.com. 2014 [cited 2020 Aug 29]. Available from: https://www.uspharmacist.com/article/trends-in-m rsaprevalence

[8] Kourtis AP. Vital signs: Epidemiology and recent trends in methicillinresistant and in methicillin-susceptible Staphylococcus aureus bloodstream infections - United States. MMWR Morb Mortal Wkly Rep [Internet]. 2019 [cited $2020 \quad$ Aug 30];68. Available from: https://www.cdc.gov/mmwr/volumes/68/wr/mm6809e1.htm

[9] WHO publishes list of bacteria for which new antibiotics are urgently needed [Internet]. Who.int. [cited 2020 Aug 29]. Available from: https://www.who. int/news-room/detail/27-02-2017-who-publishes-listof-bacteria-for-which-new-antibiotics-are-urgently-needed 
[10] Gould IM, Reilly J, Bunyan D, Walker A. Costs of healthcare-associated methicillin-resistant Staphylococcus aureus and its control. Clin Microbiol Infect. 2010;16(12):1721-8. https://doi.org/10.1111/j.1469 0691.2010.03365.x

[11] Gericke CA, Britain K, Elmahdawy M, Elsisi G. Health System in Egypt. In: Health Services Research. Boston, MA: Springer US; 2018. p. 1-18. https://doi.org/10.1007/978-1-4614-6419-8_7-1

[12] MRSA in Egypt: Summary of few published reports - Outbreak News Today [Internet]. Outbreaknewstoday.com. 2017 [cited 2020 Aug 30]. http://outbreaknewstoday.com/mrsa-egypt-summary-published-reports29180/

[13] Ahmed EF, Gad GFM, Abdalla AM, Hasaneen AM, Abdelwahab SF. Prevalence of methicillin resistant Staphylococcus aureus among Egyptian patients after surgical interventions. Surg Infect (Larchmt) [Internet]. 2014;15:404-11. https://doi.org/10.1089/sur.2013.212

[14] Egypt. World health organization - eastern Mediterranean region [Internet]. Who.int. [cited 2020 Aug 30]. Available from: http://www.emro. who.int/egy/egypt-events/a-continuing-successfulcollaboration-between-who-and-health-sector-in-egypt. html

[15] Multilocus sequence typing (MLST) [Internet]. Pubmlst.org. [cited 2020 Sep 5]. Available from: https://pubmlst.org/general.shtml

[16] Oliver JD, Jones JL. Chapter 66 - Vibrio parahaemolyticus and Vibrio vulnificus. In: Tang Y-W, Sussman M, Liu D, Poxton I, Schwartzman J, editors. Molecular Medical Microbiology (Second Edition). San Diego, CA: Academic Press; 2015. p. 1169-86. https://doi.org/10.1016/B978-0-12-3971692.00066-4

[17] Raina V, Nayak T, Ray L, Kumari K, Suar M. Chapter 9 - A polyphasic taxonomic approach for designation and description of novel microbial species. In: Das S, Dash HR, editors. Microbial Diversity in the Genomic Era. San Diego, CA: Academic Press; 2019. p. 137-52. https://doi.org/10.1016/B978-0-12-814849-5.00009-5

[18] Gaiarsa S, Batisti Biffignandi G, Esposito EP, Castelli M, Jolley KA, Brisse S, et al. Comparative analysis of the two Acinetobacter baumannii multilocus sequence typing (MLST) schemes. Front Microbiol. 2019;10:930. https://doi.org/10.3389/fmicb. 2019.00930

[19] Ibarz Pavón AB, Maiden MCJ. Multilocus sequence typing. Methods Mol Biol. 2009;551:129-40. https://doi.org/10.1007/978-1-60327-999-4_11

[20] Nakaminami H, Ito A, Sakanashi D, Suematsu H, Yamagishi Y, Mikamo H, et al. Genetic diversity of pvl-positive community-onset methicillin-resistant Staphylococcus aureus isolated at a university hospital in Japan. J Infect Chemother. https://doi.org/10.1016/j.jiac. 2017.06.002

2017;23(12):856-8.

21] Wan T-W, Khokhlova OE, Iwao Y, Higuchi W, Hung W-C, Reva IV, et al. Complete circular genome sequence of successful ST8/SCCmeclV community-associated methicillin-resistant Staphylococcus aureus (OC8) in Russia: One-megabase genomic inversion, IS256's spread, and evolution of Russia ST8-IV. PLOS One. 2016;11(10):e0164168. https://doi.org/10.1371/journal. pone.0164168

[22] Becker K, Ballhausen B, Kahl BC, Köck R. The clinical impact of livestockassociated methicillin-resistant Staphylococcus aureus of the clonal complex 398 for humans. Vet Microbiol. 2017;200:33-8. https://doi.org/10.1016/j.vetmic. 2015.11.013

[23] Benito D, Aspiroz C, Gilaberte Y, Sanmartín R, Hernández-Martin Á, Alonso $M$, et al. Genetic lineages and antimicrobial resistance genotypes in Staphylococcus aureus from children with atopic dermatitis: detection of clonal complexes CC1, CC97 and CC398. J Chemother. 2016;28(5):359-66. https://doi.org/10.1179/1973947815Y.0000000044

[24] Cuny C, Witte W. MRSA in equine hospitals and its significance for infections in humans. Vet Microbiol. 2017;200:59-64. https://doi.org/10.1016/j.vetmic.2016.01.013

[25] Magro G, Rebolini M, Beretta D, Piccinini R. Methicillin-resistant Staphylococcus aureus CC22-MRSA-IV as an agent of dairy cow intramammary infections. Vet Microbiol. 2018;227:29-33. https://doi.org/10.1016/j.vetmic. 2018.10.021

[26] Chen C-J, Lauderdale T-LY, Lu C-T, Chuang Y-Y, Yang C-C, Wu T-S, et al. Clinical and molecular features of MDR livestock-associated MRSA ST9 with staphylococcal cassette chromosome mecXII in humans. J Antimicrob Chemother. 2018;73(1):33-40. https://doi.org/10.1093/jac/dkx357

[27] Groom AV, Wolsey DH, Naimi TS, Smith K, Johnson S, Boxrud D, et al. Community-acquired methicillin-resistant Staphylococcus aureus in a rural American Indian community. JAMA. 2001;286(10):1201-5. https://doi.org/10.1001/jama.286.10.1201

[28] Cuny C, Wieler LH, Witte W. Livestock-associated MRSA: The impact on humans. Antibiotics (Basel). 2015;4(4):521-43. https://doi.org/10.3390/antibiotics4040521

[29] Abolghait SK, Fathi AG, Youssef FM, Algammal AM. Methicillin-resistant Staphylococcus aureus (MRSA) isolated from chicken meat and giblets often produces staphylococcal enterotoxin $B$ (SEB) in non-refrigerated raw chicken livers. Int J Food Microbiol. 2020;328(108669):108669. https://doi.org/10.1016/j.ijfoodmicro.2020.108669

[30] Senok A, Somily A, Slickers P, Raji M, Garaween G, Shibl A, et al. Investigating a rare methicillin-resistant Staphylococcus aureus strain: first description of genome sequencing and molecular characterization of CC15MRSA. Infect Drug Resist. 2017;10:307-15. https://doi.org/10.2147/IDR.S145394

[31] Senok A, Somily A, Raji A, Gawlik D, Al-Shahrani F, Baqi S, et al. Diversity of methicillin-resistant Staphylococcus aureus CC22-MRSA-IV from Saudi Arabia and the Gulf region. Int J Infect Dis. 2016;51:31-5. https://doi.org/10.1016/j. ijid.2016.08.016

[32] Benito D, Gómez P, Aspiroz C, Zarazaga M, Lozano C, Torres C. Molecular characterization of Staphylococcus aureus isolated from humans related to a livestock farm in Spain, with detection of MRSA-CC130 carrying mecC gene: A zoonotic case? Enferm Infecc Microbiol Clin. 2016b;34:280-5. https://doi.org/10.1016/j.eimc. 2015.03.008

[33] Lahuerta-Marin A, Guelbenzu-Gonzalo M, Pichon B, Allen A, Doumith M, Lavery JF, et al. First report of lukM-positive livestock-associated methicillin-resistant Staphylococcus aureus CC30 from fattening pigs in Northern Ireland. Vet Microbiol. 2016;182:131-4. https://doi. org/10.1016/j.vetmic. 2015.11.019

[34] Weese, J.S., B.P. Avery, and R.J. Reid-Smith, Detection and quantification of methicillin-resistant Staphylococcus aureus (MRSA) clones in retail meat products. Lett Appl Microbiol, 2010. 51: p. 338-42. https://doi.org/10.1111/j.1472-765X.2010.02901.x

[35] Sharma M, Nunez-Garcia J, Kearns AM, Doumith M, Butaye PR, Argudín MA, et al. Livestock-associated methicillin resistant Staphylococcus aureus (LA-MRSA) clonal complex (CC) 398 isolated from UK animals belong to European lineages. Front Microbiol. 2016;7:1741. https://doi.org/10.3389/fmicb.2016.01741

[36] Feltrin F, Alba P, Kraushaar B, lanzano A, Argudín MA, Di Matteo P, et al. A livestock-associated, multidrug-resistant, methicillin-resistant Staphylococcus aureus clonal complex 97 lineage spreading in dairy cattle and pigs in Italy. Appl Environ Microbiol. 2016;82(3):816-21. https://doi.org/10.1128/AEM.02854-15

[37] Nworie A, Onyema AS, Okekpa SI, Elom MO, Umoh NO, Usanga VU, et al. A novel methicillin-resistant Staphylococcus aureus t11469 and a poultry endemic strain t002 (ST5) are present in chicken in Ebonyi State, Nigeria. Biomed Res Int. 2017;2017:1-5. https://doi.org/10.1155/2017/2936461

[38] Butaye P, Argudín MA, Smith TC. Livestock-associated MRSA and its current evolution. Curr Clin Microbiol Rep. 2016;3(1):19-31. https://doi.org/10.1007/s40588-016-0031-9

[39] Abd El-Hamid MI, Bendary MM, Merwad AMA, Elsohaby I, Mohammad Ghaith D, Alshareef WA. What is behind phylogenetic analysis of hospital-, community- and livestock-associated methicillin-resistant Staphylococcus aureus? Transbound Emerg Dis. 2019;66(4):1506-17. https://doi.org/10.1111/tbed.13170

[40] Ballhausen B, Kriegeskorte A, van Alen S, Jung P, Köck R, Peters G, et al. The pathogenicity and host adaptation of livestock-associated MRSA CC398. Vet Microbiol. 2017;200:39-45 https://doi.org/10.1016/j.vetmic. 2016.05.006

[41] Mutters NT, Bieber CP, Hauck C, Reiner G, Malek V, Frank U. Comparison of livestock-associated and health care-associated MRSA-genes, virulence, and resistance. Diagn Microbiol Infect Dis. 2016;86:417-21. https://doi.org/10.1016/j.diagmicrobio.2016.08.016 
[42] Rolo J, Worning P, Boye Nielsen J, Sobral R, Bowden R, Bouchami O, et al. Evidence for the evolutionary steps leading to mecA-mediated $\beta$-lactam resistance in staphylococci. PLoS Genet. 2017;13(4):e1006674. https://doi. org/10.1371/journal.pgen.1006674

[43] Beims $H$, Overmann A, Fulde $M$, Steinert $M$, Bergmann S. Isolation of Staphylococcus sciuri from horse skin infection. Open Vet J. 2016;6(3):2426. https://doi.org/10.4314/ovj.v6i3.14

[44] Zhou W, Li X, Osmundson T, Shi L, Ren J, Yan H. WGS analysis of ST9-MRSA$\mathrm{XII}$ isolates from live pigs in China provides insights into transmission among porcine, human and bovine hosts. J Antimicrob Chemother. 2018;73:265261. https://doi.org/10.1093/jac/dky245

[45] Sahibzada S, Abraham S, Coombs GW, Pang S, Hernández-Jover M, Jordan D, et al. Transmission of highly virulent community-associated MRSA ST93 and livestock-associated MRSA ST398 between humans and pigs in Australia. Sci Rep. 2017;7:5273. https://doi.org/10.1038/s41598-01704789-0

[46] Kluytmans, J., et al., Food-initiated outbreak of methicillin-resistant Staphylococcus aureus analyzed by pheno- and genotyping. J Clin Microbiol, 1995. 33(5): p. 1121-8. https://doi.org/10.1128/JCM.33.5.1121-1128.1995

[47] Zayda MG, Masuda Y, Hammad AM, Honjoh K-I, Elbagory AM, Miyamoto T. Molecular characterisation of methicillin-resistant (MRSA) and methicillin-susceptible (MSSA) Staphylococcus aureus isolated from bovine subclinical mastitis and Egyptian raw milk cheese. Int Dairy J. 2020;104 (104646):104646. https://doi.org/10.1016/j.idairyj.2020.104646

[48] Humphreys $\mathrm{H}$, Coleman DC. Contribution of whole-genome sequencing to understanding of the epidemiology and control of meticillin-resistant Staphylococcus aureus. J Hosp Infect. 2019;102:189-99. https://doi.org/10.1016/j.jhin.2019.01.025

[49] Madigan T, Cunningham SA, Patel R, Greenwood-Quaintance KE, Barth JE, Sampathkumar $P$, et al. Whole-genome sequencing for methicillin-resistant Staphylococcus aureus (MRSA) outbreak investigation in a neonatal intensive care unit. Infect Control Hosp Epidemiol. 2018;39:1412-8. https://doi.org/10.1017/ice.2018.239 